\title{
INVARIANCE ENTROPY FOR TOPOLOGICAL SEMIGROUP ACTIONS
}

\author{
FRITZ COLONIUS, RYUICHI FUKUOKA, AND ALEXANDRE J. SANTANA
}

\author{
(Communicated by Yingfei Yi)
}

\begin{abstract}
Invariance entropy for the action of topological semigroups acting on metric spaces is introduced. It is shown that invariance entropy is invariant under conjugations and a lower bound and upper bounds of invariance entropy are obtained. The special case of control systems is discussed.
\end{abstract}

\section{INTRODUCTION}

Our aim is to introduce and to study a certain invariant for the action of topological semigroups on metric spaces. This is motivated by recent work on invariance entropy for control systems. The original idea to use concepts close to topological entropy for control systems is due to Nair, Evans, Mareels and Moran [15], who studied feedback entropy in an engineering context. A related notion, called invariance entropy, has been considered by Colonius and Kawan in [6]. It has proved to be a fruitful approach for the study of control systems; cf. Kawan [11 13. On the other hand, Hoffmann and Stojanov in 8 introduced a notion of topological entropy for semigroup actions. Although topological entropy is rather different from invariance entropy, we show in the present note that one can combine some of their concepts, in particular, regular systems with the constructions of invariance entropy. This results in a notion of (topological) invariance entropy for semigroup actions.

The content of this note is the following. Section 1 introduces weakly almost invariant sets for topological semigroup actions on metric spaces and defines a corresponding invariance entropy. It is shown that this number is an invariant under appropriately defined topological conjugacies. Section 2 gives upper bounds and a lower bound for invariance entropy. Section 3 shows that for control systems, the notion considered here is equivalent to the invariance entropy from [6]. Here the main work consists of showing that arbitrary admissible families can be approximated by admissible families defined via controls.

We also remark that the notion of topological entropy for maps on noncompact spaces (in particular for automorphisms of Lie groups) studied by M. Patrao [16]

Received by the editors August 11, 2011 and, in revised form, February 12, 2012.

2010 Mathematics Subject Classification. Primary 54H15; Secondary 37B40, 93C25.

Key words and phrases. Invariance entropy, semigroup actions, weak invariance.

The research of the first author was partially supported by DFG grant Co 124/17-2 within DFG Priority Program 1305 Control Theory of Digitally Networked Dynamical Systems.

The research of the second author was partially supported by the CNPq Grant 305557/2009-2.

The research of the third author was partially supported by the Fundação Araucária Grant $496 / 10$. 
is different from the one in [8]. For connections between semigroup actions and control we refer to San Martin [17.

Among the many problems left open in the present note is an Abramov-type theorem: what happens if we consider a subsemigroup, say, with compact left cosets?

\section{WEAK INVARIANCE AND INVARIANCE ENTROPY}

In this section we introduce regular systems and admissible families for semigroup actions. Then we define an invariance entropy for weakly almost invariant sets.

Let $(S, \cdot)$ be a topological semigroup acting on a metric space $M$; i.e., there is a continuous map

$$
\varphi: M \times S \rightarrow M,(x, s) \mapsto \varphi(x, s)=x \cdot s
$$

satisfying $x \cdot e=x$ for the neutral element $e \in S$ and $\left(x \cdot s_{1}\right) \cdot s_{2}=x \cdot\left(s_{1} \cdot s_{2}\right)$ for all $x \in M$ and $s_{1}, s_{2} \in S$.

First, we slightly modify the notion of regular systems introduced by Hoffmann and Stojanov [8].

Definition 1.1. A family of subsets $A_{\tau} \subset S, \tau \in[0, \infty)$, is called a regular system if every set $A_{\tau}$ contains the neutral element $e$ and

$$
A_{\tau} \cdot A_{\sigma} \subset A_{\tau+\sigma} \text { for } \tau, \sigma \in[0, \infty) .
$$

We also abbreviate $A_{<\tau}:=\bigcup_{\sigma \in[0, \tau)} A_{\sigma}$ for $\tau>0$.

The following definition of admissible (one-parameter) families is crucial.

Definition 1.2. A map $\gamma: I \rightarrow S$ with $I:=[0, \infty)$ or $I:=\left[0, t_{1}\right], t_{1}>0$, is called an admissible family in the semigroup $S$ if

(i) the map $(x, \tau) \mapsto x \cdot \gamma(\tau): M \times I \rightarrow M$ is continuous;

(ii) for all $\sigma, \tau \geq 0$ with $\sigma+\tau \in I$ there is $s \in A_{\tau} \backslash A_{<\tau}$ with $\gamma(\sigma) \cdot s=\gamma(\sigma+\tau)$ and $\gamma(0)=e$.

Throughout the rest of this paper, we keep a regular system $\left(A_{\tau}\right)_{\tau \in[0, \infty)}$ in a semigroup $S$ acting on $M$ fixed. Note the following comments on these definitions.

Remark 1.3. For this definition of regular systems compare the notions of regular systems and one-parameter semigroups of compact sets by Hofmann and Stojanov [8. Among other things, our definition of regular systems does not require that $\bigcup_{\tau>0} A_{\tau}=S$. This property would entail a closer connection between the regular system and the semigroup.

Remark 1.4. Note that condition (ii) in Definition 1.2 with $\sigma=0$ implies that for all $\tau>0$ one has $\gamma(\tau)=\gamma(0) \cdot s=s \in A_{\tau} \backslash A_{<\tau}$. An immediate consequence is that the regular family is strictly increasing with $\tau$ if there is an admissible family. Furthermore, the trivial map $\gamma(t) \equiv e$ does not define an admissible family.

Remark 1.5. One could also specify subclasses of admissible families. We will follow this path only in Theorem 2.4, where we impose a Lipschitz condition.

An example of a semigroup action with a regular system is given by a control system $\Sigma$ on a differentiable manifold $M$ of the form

$$
\dot{x}(t)=f(x(t), u(t)),
$$


where $u$ is in a set $\mathcal{U}$ of control functions defined on $[0, \infty)$ with values in a set $U$ and $f(x(t), u(t))$ is a vector in the tangent space $T_{x(t)} M$ of $M$ at $x(t)$ (see Agrachev and Sachkov [2]). We assume that $\mathcal{U}$ is shift invariant; i.e., the classical shift function $\Theta: \mathbb{R}_{+} \times \mathcal{U} \rightarrow \mathcal{U},(t, u) \mapsto u(t+\cdot)$ is well defined. For a fixed $u \in \mathcal{U}$, we denote the solution of (1.2) with initial condition $x(0)=x_{0}$ by $\varphi\left(t, x_{0}, u\right), t \geq 0$. We suppose that unique global solutions exist for all controls $u \in \mathcal{U}$. Define the semigroup

$$
S:=\{(t, \varphi(t, \cdot, u)), t \in[0, \infty) \text { and } u \in \mathcal{U}\}
$$

with the semigroup operation given by

$$
\left(t_{1}, \varphi\left(t_{1}, \cdot, u_{1}\right)\right) \cdot\left(t_{2}, \varphi\left(t_{2}, \cdot, u_{2}\right)\right):=\left(t_{1}+t_{2}, \varphi\left(t_{1}+t_{2}, \cdot, u_{1} \&_{t_{1}} u_{2}\right)\right)
$$

where $u_{1} \&_{t_{1}} u_{2}$ stands for the concatenation

$$
\left(u_{1} \&_{t_{1}} u_{2}\right)(t):= \begin{cases}u_{1}(t) & \text { if } t \in\left[0, t_{1}\right] \\ u_{2}\left(t-t_{1}\right) & \text { if } t>t_{1}\end{cases}
$$

Here we also assume that all these concatenations again are in $\mathcal{U}$. The action is defined by $(x,(t, \varphi(t, \cdot, u))) \mapsto \varphi(t, x, u): M \times S \rightarrow M$. Observe that $\varphi\left(t_{1}+\right.$ $\left.t_{2}, x, u\right):=\varphi\left(t_{2}, \varphi\left(t_{1}, x, u\right), \Theta\left(t_{1}, u\right)\right)$ and that $\Theta\left(t_{1}, u_{1} \&_{t_{1}} u_{2}\right)=u_{2}$.

A regular system is given by

$$
A_{\tau}:=\{(t, \varphi(t, \cdot, u)) \in S, t \in[0, \tau]\}, \tau \geq 0,
$$

and it is straightforward to see that for a fixed $u \in \mathcal{U}$, the function defined by $\gamma(\tau):=(\tau, \varphi(\tau, \cdot, u)), \tau \geq 0$, is an admissible family. Note that not every admissible family is of this form, since it may not correspond to a single control.

Remark 1.6. Often one associates a semigroup to a control system by considering the diffeomorphisms associated to piecewise constant controls (cf. Jurdjevic [9] or Agrachev and Sachkov [2]). We use the alternative construction above, since we do not want to restrict our attention to these special controls (locally integrable controls are a more natural choice). Furthermore, the time $t$ is added in the semigroup (1.3) in order to guarantee that we obtain admissible families.

Now we can introduce our notion of invariance.

Definition 1.7. Consider a semigroup action (1.1) endowed with a regular system $\left(A_{\tau}\right)_{\tau \geq 0}$. A subset $Q \subset M$ is called weakly almost invariant if for every $x \in Q$, $\varepsilon>0$ and $\tau>0$, there is an admissible family $\gamma:[0, \tau] \rightarrow S$ such that

$$
d(x \cdot \gamma(\sigma), Q):=\inf _{q \in Q}\{d(x \cdot \gamma(\sigma), q)\}<\varepsilon \text { for all } \sigma \in[0, \tau] .
$$

Next we define invariance entropy in the following steps.

Definition 1.8. Consider the semigroup action (1.1) endowed with a regular system $\left(A_{\tau}\right)_{\tau \in[0, \infty)}$. Let $Q \subset M$ be a weakly almost invariant set and consider a subset $K \subset Q$. For $\varepsilon>0$ and $\tau>0$ a set $R(\tau, \varepsilon, K, Q)$ of admissible families is called $(\tau, \varepsilon, K, Q)$-spanning if for every $x \in K$ there exists $\gamma \in R(\tau, \varepsilon, K, Q)$ such that $d(x \cdot \gamma(t), Q)<\varepsilon$ for all $t \in[0, \tau]$.

There are always finite $(\tau, \varepsilon, K, Q)$-spanning sets.

Lemma 1.9. Let $K$ be a compact subset of a weakly almost invariant set $Q \subset M$ and let $\tau, \varepsilon>0$. Then there exists a finite $(\tau, \varepsilon, K, Q)$-spanning set. 
Proof. Let $x \in K$. Then there exists an admissible family $\gamma_{x}$ such that $d(x$. $\left.\gamma_{x}(t), Q\right)<\varepsilon$ for all $t \in[0, \tau]$. By continuity and compactness of $[0, \tau]$, all $y$ in a neighborhood of $x$ satisfy $d\left(y \cdot \gamma_{x}(t), Q\right)<\varepsilon$ for all $t \in[0, \tau]$. Then compactness of $K$ shows that finitely many admissible families form a $(\tau, \varepsilon, K, Q)$-spanning family.

This lemma justifies the following definition, which is the central notion of the present note.

Definition 1.10. Consider the semigroup action $S$ on $M$ of the form (1.1) endowed with a regular system $\left(A_{\tau}\right)_{\tau \in[0, \infty)}$. Let $K$ be a compact subset of a weakly almost invariant set $Q \subset M$. We denote the minimal cardinality of a $(\tau, \varepsilon, K, Q)$-spanning set by $r_{i n v}^{S}(\tau, \varepsilon, K, Q)$. The invariance entropy of $(K, Q)$ is defined by

$$
\begin{aligned}
h_{i n v}^{S}(\varepsilon, K, Q) & :=\limsup _{\tau \rightarrow \infty} \frac{1}{\tau} \log r_{i n v}^{S}(\tau, \varepsilon, K, Q), \\
h_{i n v}^{S}(K, Q) & :=\lim _{\varepsilon \rightarrow 0} h_{i n v}^{S}(\varepsilon, K, Q) .
\end{aligned}
$$

Note that the limit for $\varepsilon \rightarrow 0$ exists, since for $\varepsilon_{1}>\varepsilon_{2}>0$ every $\left(\tau, \varepsilon_{2}, K, Q\right)$ spanning set is also $\left(\tau, \varepsilon_{1}, K, Q\right)$-spanning, implying that $h_{i n v}^{S}(\varepsilon, K, Q)$ is monotonically increasing for $\varepsilon \rightarrow 0$.

Remark 1.11. One can also define weakly invariant sets by requiring that for every $x \in Q$ and $\tau>0$ there is an admissible family $\gamma$ with $x \cdot \gamma(\sigma) \in Q$ on $[0, \tau]$. An associated notion of strict invariance entropy $h_{\text {inv }}^{S, *}(K, Q)$ can be based on the exponential growth rate of the minimal number $r_{i n v}^{S, *}(\tau, K, Q)$ of spanning admissible families of this type. Obviously one has

$$
h_{\text {inv }}^{S, *}(K, Q) \geq h_{\text {inv }}^{S}(K, Q) .
$$

However, in general, we cannot guarantee that $r_{i n v}^{S}(\tau, K, Q)$ is finite (but see Theorem 2.3. and also Kawan 12 for results on strict invariance entropy in the controltheoretic context).

In Section 3 we will show that for control systems of the form (1.3) the notion of invariance entropy for semigroup actions coincides with the one considered in Colonius and Kawan [6].

Next we introduce a notion of topological conjugation and study the behavior of invariance entropy under these conjugations. A homomorphism $\pi_{S}: S^{1} \rightarrow S^{2}$ of semigroups maps the neutral element $e^{1} \in S^{1}$ to the neutral element $e^{2} \in S^{2}$ and $\pi_{S}\left(s \cdot s^{\prime}\right)=\pi_{S}(s) \cdot \pi_{S}\left(s^{\prime}\right)$ for all $s, s^{\prime} \in S^{1}$.

Definition 1.12. For $i=1,2$, let $\varphi^{i}: M^{i} \times S^{i} \rightarrow M^{i}$ be semigroup actions with regular systems $A^{i}=\left\{A_{\tau}^{i}, \tau \geq 0\right\}$. Let $\pi_{M}: M^{1} \rightarrow M^{2}$ be a continuous map and let $\pi_{S}: S^{1} \rightarrow S^{2}$ be a semigroup homomorphism such that

$$
\pi_{M}(x \cdot s)=\pi_{M}\left(\varphi^{1}(x, s)\right)=\varphi^{2}\left(\pi_{M}(x), \pi_{S}(s)\right)=\pi_{M}(x) \cdot \pi_{S}(s)
$$

for all $(s, x) \in S^{1} \times M^{1}$. Suppose, furthermore, that for all $\tau \geq 0$,

$$
\pi_{S}\left(A_{\tau}^{1}\right) \subset A_{\tau}^{2} \text { and }\left(\pi_{S}\right)^{-1}\left(A_{\tau}^{2}\right) \subset A_{\tau}^{1} .
$$

In this situation we say that the semigroup action $\varphi^{1}$ is semiconjugate to $\varphi^{2}$. If $\pi_{M}$ is a homeomorphism and $\pi_{S}$ is bijective, we say that these semigroup actions are conjugate.

Next we show that conjugation preserves the invariance entropy. 
Theorem 1.13. Let $\varphi^{1}: M^{1} \times S^{1} \rightarrow M^{1}$ and $\varphi^{2}: M^{2} \times S^{2} \rightarrow M^{2}$ be two semigroup actions with regular systems $A^{1}$ and $A^{2}$, respectively. Assume that there exists a semiconjugation $\left(\pi_{M}, \pi_{S}\right)$ from $\varphi^{1}$ to $\varphi^{2}$. Let $K \subset Q \subset M^{1}$ and suppose that $K$ is compact and $Q$ is weakly almost invariant. If $\pi_{M}$ is uniformly continuous on a $\delta$ neighborhood of $Q$, then $\pi_{M}(Q)$ is weakly almost invariant and $h_{i n v}(\pi(K), \pi(Q)) \leq$ $h_{\text {inv }}(K, Q)$.

In particular, if the semigroup actions are conjugate, the invariance entropy is preserved.

Proof. It is clear that $\pi_{M}(K) \subset \pi_{M}(Q)$ are nonvoid sets and that $\pi_{M}(K)$ is compact. First we show that $\pi_{M}(Q)$ is weakly almost invariant for the semigroup action $\varphi^{2}$. In fact, this follows from equation (1.7): If $y \in \pi_{M}(Q)$, there is $x \in Q$ with $y=\pi_{M}(x)$. Let $\varepsilon>0$ and $\tau>0$. Since $\pi_{M}$ is uniformly continuous on a neighborhood of $Q$ there exists $\delta>0$ with $\pi_{M}\left(\mathbf{N}_{\delta}(Q)\right) \subset \mathbf{N}_{\varepsilon}\left(\pi_{M}(Q)\right)$, where $\mathbf{N}_{\varepsilon}(Q)$ denotes the set of points with distance less than $\varepsilon$ to $Q$. Let $\gamma:[0, \tau] \rightarrow S^{1}$ be an admissible family such that $d(x \cdot \gamma(t), Q)<\delta$ for all $t \in[0, \tau]$. Then by (1.7)

$$
\pi_{M}(x) \cdot \pi_{S}(\gamma(t))=\pi_{M}(x \cdot \gamma(t)) \in \pi_{M}\left(\mathbf{N}_{\delta}(Q)\right) \subset \mathbf{N}_{\varepsilon}\left(\pi_{M}(Q)\right),
$$

and weak almost invariance of $\pi_{M}(Q)$ follows. Now let $\tau, \varepsilon>0$. As above, there exists $\delta>0$ with $\pi_{M}\left(\mathbf{N}_{\delta}(Q)\right) \subset \mathbf{N}_{\varepsilon}\left(\pi_{M}(Q)\right)$. Let $R \subset S^{1}$ be a minimal $(\tau, \delta, K, Q)$ spanning set of admissible families in $A^{1}$ and consider $\pi_{S}(R)$. For any $y \in \pi_{M}(K)$ there exists $x \in K$ with $\pi_{M}(x)=y$. Let $\gamma \in R$ such that $d(x \cdot \gamma(t), Q)<\delta$ for all $t \in[0, \tau]$. Then $\pi_{M}(x) \cdot \pi_{S}(\gamma(t)) \in \pi_{M}\left(\mathbf{N}_{\delta}(Q)\right) \subset \mathbf{N}_{\varepsilon}\left(\pi_{M}(Q)\right)$.

Furthermore, all maps $\pi_{S} \circ \gamma \in \pi_{S}(R)$ satisfy the conditions on admissible families in Definition 1.2. This is clear for (i), and using (1.7) one finds for $\sigma, \tau \geq 0$ an element $s \in A_{\tau}^{1} \backslash A_{<\tau}^{1}$ with

$$
\pi_{M}(\gamma(\sigma)) \cdot \pi_{S}(s)=\pi_{M}(\gamma(\sigma) \cdot s)=\pi_{M}(\gamma(\sigma+\tau))=\left(\pi_{M} \circ \gamma\right)(\sigma+\tau) .
$$

By the first condition in (1.8) one has $\pi_{S}(s) \in A_{\tau}^{2}$. If $\pi_{S}(s) \in A_{\sigma}^{2}$ for some $\sigma<\tau$, the second condition in (1.8) yields the contradiction $s \in\left(\pi_{S}\right)^{-1}\left(A_{\sigma}^{2}\right) \subset A_{\sigma}^{1}$. This shows property (ii). It follows that $\pi_{S}(R)$ is an admissible family for $\varphi^{2}$. Consequently the minimal cardinality of a $\left(\tau, \varepsilon, \pi_{M}(K), \pi_{M}(Q)\right)$-spanning set is not greater than the cardinality of $R$, and hence

$$
h_{i n v}(\varepsilon, \pi(K), \pi(Q)) \leq h_{i n v}(\delta, K, Q) \leq h_{i n v}(K, Q) .
$$

For $\varepsilon \rightarrow 0$, the assertion follows.

Remark 1.14. It is not difficult to see that the invariance entropy is not an invariant under the more general topological conjugacies considered in Ayala et al. 3] and Baratchart et al. [4]. These conjugacies, which are defined for skew product flows resulting from control systems, would, in our framework, allow that the homeomorphism $\pi_{M}$ may also depend on the semigroup elements.

\section{UPPER AND LOWER BOUNDS FOR INVARIANCE ENTROPY}

In this section we will prove upper bounds and a lower bound for invariance entropy.

Definition 2.1. If $\gamma_{1}:\left[0, t_{1}\right] \rightarrow S$ and $\gamma_{2}:\left[0, t_{2}\right] \rightarrow S$ are two admissible families in a semigroup $S$, then the concatenation $\gamma_{1} \cdot \gamma_{2}:\left[0, t_{1}+t_{2}\right] \rightarrow S$ is defined by

$$
\left(\gamma_{1} \cdot \gamma_{2}\right)(t)=\left\{\begin{array}{lll}
\gamma_{1}(t) & \text { if } & t \in\left[0, t_{1}\right] \\
\gamma_{1}\left(t_{1}\right) \cdot \gamma_{2}\left(t-t_{1}\right) & \text { if } \quad t \in\left(t_{1}, t_{1}+t_{2}\right]
\end{array}\right.
$$


The concatenation is associative, but it does not necessarily give an admissible family. In Theorem 2.3 we suppose that concatenations of admissible families again form an admissible family. The following proposition shows that this is satisfied under an additional assumption for the regular system, which for instance is satisfied for control systems of the form (1.2) with regular system given by (1.4).

Proposition 2.2. Suppose that the regular family satisfies $\left(A_{\sigma} \backslash A_{<\sigma}\right) \cdot\left(A_{\tau} \backslash A_{<\tau}\right)$ $\subset A_{\sigma+\tau} \backslash A_{<\sigma+\tau}$ for all $\sigma, \tau>0$. Then the concatenation $\gamma:=\gamma_{1} \cdot \gamma_{2}$ of admissible families $\gamma_{1}:\left[0, t_{1}\right] \rightarrow S$ and $\gamma_{2}:\left[0, t_{2}\right] \rightarrow S$ again is an admissible family.

Proof. It is clear that the continuity condition (i) in Definition 1.2 is satisfied. Condition (ii) is certainly satisfied if $\sigma+\tau \in\left[0, t_{1}\right]$. Hence we may suppose $\sigma+\tau>$ $t_{1}$. If $\sigma>t_{1}$, one finds $s \in A_{\tau} \backslash A_{<\tau}$ such that

$$
\left(\gamma_{1} \cdot \gamma_{2}\right)(\sigma+\tau)=\gamma_{1}\left(t_{1}\right) \cdot \gamma_{2}\left(\sigma+\tau-t_{1}\right)=\gamma_{1}\left(t_{1}\right) \cdot \gamma_{2}\left(\sigma-t_{1}\right) \cdot s=\gamma(\sigma) \cdot s .
$$

If $\sigma \in\left[0, t_{1}\right)$ one finds $s_{1} \in A_{t_{1}-\sigma} \backslash A_{<t_{1}-\sigma}$ such that with $s:=s_{1} \cdot \gamma_{2}\left(\sigma+\tau-t_{1}\right)$,

$$
\left(\gamma_{1} \cdot \gamma_{2}\right)(\sigma+\tau)=\gamma_{1}\left(t_{1}\right) \cdot \gamma_{2}\left(\sigma+\tau-t_{1}\right)=\gamma_{1}(\sigma) \cdot s_{1} \cdot \gamma_{2}\left(\sigma+\tau-t_{1}\right)=\gamma(\sigma) \cdot s .
$$

Taking into account Remark 1.4 one sees that

$$
s=s_{1} \cdot \gamma_{2}\left(\sigma+\tau-t_{1}\right) \in\left(A_{t_{1}-\sigma} \backslash A_{<t_{1}-\sigma}\right) \cdot\left(A_{\sigma+\tau-t_{1}} \backslash A_{<\sigma+\tau-t_{1}}\right) \subset A_{\tau} \backslash A_{<\tau} .
$$

The same remark shows the assertion for $\sigma=t_{1}$.

Recall the definition of weakly invariant sets and of strict invariance entropy in Remark 1.11.

Theorem 2.3. Let $\varphi: M \times S \rightarrow M$ be a semigroup action with regular system $\left\{A_{\tau}\right\}_{\tau \geq 0}$. Let $K \subset M$ be a compact subset and suppose that $K=\bigcup_{i=1}^{N} C_{i}$ with admissible families $\gamma_{i}:\left[0, T_{i}\right] \rightarrow S, T_{i}>0, i=1, \ldots, N$, such that $C_{i} \cdot \gamma_{i}(t) \in K$ for every $t \in\left[0, T_{i}\right]$. Assume, furthermore, that any (repeated) concatenation of the $\gamma_{i}$ again yields an admissible family. Then $K$ is a weakly invariant subset and the strict invariance entropy satisfies

$$
h_{\text {inv }}^{S, *}(K, K) \leq \frac{\log N}{\min _{i} T_{i}} .
$$

Proof. Let $T:=\min _{i=1, \ldots, N} T_{i}$. For $\tau>0$ there are $k \in \mathbb{N}$ and $\sigma \in(0, T]$ with $\tau=(k-1) T+\sigma$. We claim that for every $x \in K$, there exists an $k$-tuple $\left(i_{1}, \ldots, i_{k}\right) \in$ $\{1, \ldots, N\}^{k}$ such that $x \cdot\left(\gamma_{i_{1}} \cdot \gamma_{i_{2}} \cdot \ldots \cdot \gamma_{i_{k}}\right)(t) \in K$ for every $t \in[0, k T]$. In fact, if $x \in C_{i_{1}}$, notice that $x \cdot \gamma_{i_{1}}(t) \in K$ for every $t \in\left[0, T_{i_{1}}\right]$. Now $x \cdot \gamma_{i_{1}}\left(T_{i_{1}}\right) \in C_{i_{2}}$ for some $i_{2} \in\{1, \ldots, N\}$ and $x \cdot\left(\gamma_{i_{1}} \cdot \gamma_{i_{2}}\right)(t) \in K$ for every $t \in\left[0, T_{i_{1}}+T_{i_{2}}\right]$. We can proceed in this way up to time $\tau \leq k T \leq T_{i_{1}}+\ldots+T_{i_{k}}$ and the claim follows. For every $k$-tuple $\left(i_{1}, \ldots, i_{k}\right) \in\{1, \ldots, N\}^{k}$, the concatenation $\gamma_{i_{1}} \cdot \ldots \cdot \gamma_{i_{k}}$ is an admissible family. Moreover, the set $K$ is weakly invariant and

$$
r_{i n v}^{S, *}(\tau, 0, K, K) \leq r_{i n v}^{S, *}(k T, 0, K, K) \leq N^{k} .
$$

We conclude that

$$
h_{\text {inv }}^{S, *}(K, K)=\lim _{\tau \rightarrow \infty} \frac{1}{\tau} \log r_{\text {inv }}^{S, *}(\tau, 0, K, K) \leq \lim _{k \rightarrow \infty} \frac{1}{(k-1) T} \log N^{k}=\frac{1}{T} \log N .
$$


The assumptions of Theorem 2.3 guarantee finiteness of the strict invariance entropy $h_{i n v}^{S, *}(K, K)$ which is an upper bound for the invariance entropy $h_{i n v}^{S}(K, K)$; cf. Remark 1.11] In the following, we prove an upper bound for the invariance entropy under weaker assumptions. We will use arguments similar to Katok and Hasselblatt [10, Theorem 3.2.9] establishing an upper bound for the topological entropy of Lipschitz continuous maps; see also 6]. In our context, we have to sharpen our assumption on invariance of $Q$ by considering Lipschitz continuous admissible families defined as follows:

For constants $L, c>0$ the set $\Gamma(L, c)$ consists of all admissible families $\gamma$ such that for all $x, y \in Q$,

$$
d(x \cdot \gamma(t), y \cdot \gamma(t))<c e^{L t} d(x, y) \text { for all } t \geq 0 .
$$

We say that a set $Q$ is weakly invariant with admissible families in $\Gamma(L, c)$ if for every $x \in Q$ and $\tau>0$ there is $\gamma \in \Gamma(L, c)$ with $x \cdot \gamma(\sigma) \in Q$ for $\sigma \in[0, \tau]$, and similarly for weak almost invariance.

Recall that the upper ball dimension of $K \subset M$ is

$$
\operatorname{dim}_{B} K:=\limsup _{\delta \searrow 0} \frac{\ln b(\delta, K)}{\ln (1 / \delta)},
$$

where $b(\delta, K)$ is the minimal cardinality of a cover of $K$ by $\delta$-balls.

Theorem 2.4. Let $\varphi: M \times S \rightarrow M$ be a semigroup action with regular system $\left\{A_{\tau}\right\}_{\tau \geq 0}$. Suppose that $K \subset Q$ is a compact subset of $M$ and suppose that $Q$ is weakly invariant with admissible families in $\Gamma(L, c)$ for some fixed $L, c>0$. Then the invariance entropy satisfies

$$
h_{i n v}^{S}(K, Q) \leq L \operatorname{dim}_{B} K .
$$

Proof. Let $\varepsilon, \tau>0$. Since $Q$ is weakly invariant one finds for every $x \in K$ an admissible family $\gamma \in \Gamma(L, c)$ with $x \cdot \gamma(t) \in Q$ for all $t \in[0, \tau]$. Consider a set $R_{0}$ of pairs $(x, \gamma)$ of points in $K$ and admissible families $\gamma \in \Gamma(L, c)$ having the property that $x \cdot \gamma(t) \in Q$ for all $t \in[0, \tau]$ and for every $y \in K$ there is $(x, \gamma) \in R_{0}$ with

$$
d(x \cdot \gamma(t), y \cdot \gamma(t))<\varepsilon \text { for all } t \in[0, \tau] .
$$

Then, by compactness of $K$ and continuity there exists such a set $R_{0}$ with finite cardinality. Choose a set $R_{0}$ with minimal cardinality $r$. Then one easily sees that $r$ is greater than or equal to the minimal cardinality $r_{i n v}^{S}(\varepsilon, \tau, K, Q)$ of a $(\tau, \varepsilon, K, Q)$ spanning set $R(\tau, \varepsilon, K, Q)$.

Define for $\left(x_{i}, \gamma_{i}\right) \in R_{0}, i=1, \ldots, r$, the sets

$$
N_{i}:=\left\{x \in K, d\left(x \cdot \gamma_{i}(t), x_{i} \cdot \gamma_{i}(t)\right)<\varepsilon \text { for all } t \in[0, \tau]\right\} .
$$

Then $K$ is contained in $\bigcup_{i=1}^{r} N_{i}$.

Let $x \in M$ be a point with $d\left(x, x_{i}\right)<e^{-L \tau} \varepsilon / c$ for some $i \in\{1, \ldots, r\}$. For all $t \in[0, \tau]$, it follows that

$$
d\left(x \cdot \gamma_{i}(t), x_{i} \cdot \gamma_{i}(t)\right)<c e^{L t} d\left(x, x_{i}\right)<\varepsilon .
$$

Hence $x \in N_{i}$, and thus $N_{i}$ contains the ball $\mathbf{B}\left(x_{i} ; e^{-L \tau} \varepsilon / c\right)$.

Next we claim that $r \leq b\left(e^{-L \tau} \varepsilon / c, K\right)$. Assume to the contrary that there exists a cover $\mathcal{C}$ of $K$ consisting of $e^{-L \tau} \varepsilon / c$-balls such that $n:=\# \mathcal{C}<r$. Let these balls be centered at points $\tilde{x}_{1}, \ldots, \tilde{x}_{n} \in Q$. By weak invariance of $Q$ we can assign to $\tilde{x}_{j}$ an 
admissible family $\tilde{\gamma}_{j}$ with $\tilde{x}_{j} \cdot \tilde{\gamma}_{j}(t) \in Q$ for all $t \geq 0$. Then the ball $\mathbf{B}\left(\tilde{x}_{j} ; e^{-L \tau} \varepsilon / c\right)$ is contained in the set

$$
V_{j}:=\left\{x \in M, d\left(x \cdot \tilde{\gamma}_{j}(t), \tilde{x}_{j} \cdot \tilde{\gamma}_{j}(t)\right)<\varepsilon \text { for all } t \in[0, \tau]\right\} .
$$

Thus the set $\left\{\left(\tilde{x}_{1}, \tilde{\gamma}_{1}\right), \ldots,\left(\tilde{x}_{n}, \tilde{\gamma}_{n}\right)\right\}$ also has the property that for every $x \in K$ there is $\left(\tilde{x}_{j}, \tilde{\gamma}_{j}\right)$ with

$$
d\left(x \cdot \tilde{\gamma}_{j}(t), \tilde{x}_{j} \cdot \tilde{\gamma}_{j}(t)\right)<\varepsilon \text { for all } t \in[0, \tau] .
$$

This contradicts the minimality of $r$. It follows that

$$
r_{i n v}^{S}(\varepsilon, \tau, K, Q) \leq r \leq b\left(e^{-L \tau} \varepsilon / c, K\right) .
$$

Observe that

$$
\ln \left(1 /\left(e^{-L \tau} \varepsilon / c\right)\right)=\ln \left(e^{L \tau} c / \varepsilon\right)=L \tau+\ln c-\ln \varepsilon .
$$

We find, with $\delta:=e^{-L \tau} \varepsilon / c \rightarrow 0$ for $\tau \rightarrow \infty$, that

$$
\begin{aligned}
\limsup _{\tau \rightarrow \infty} \frac{1}{\tau} \ln r_{i n v}^{S}(\varepsilon, \tau, K, Q) & \leq \limsup _{\tau \rightarrow \infty} \frac{1}{\tau} \ln b\left(e^{-L \tau} \varepsilon / c, K\right) \\
& =\limsup _{\tau \rightarrow \infty}\left[\frac{L \tau+\ln c-\ln \varepsilon}{\tau} \cdot \frac{\ln b\left(e^{-L \tau} \varepsilon / c, K\right)}{\ln \left(1 /\left(e^{-L \tau} \varepsilon / c\right)\right.}\right] \\
& =L \limsup _{\tau \rightarrow \infty} \frac{\ln b\left(e^{-L \tau} \varepsilon / C, K\right)}{\ln \left(1 /\left(e^{-L \tau} \varepsilon / C\right)\right)} \\
& \leq L \operatorname{dim}_{B} K .
\end{aligned}
$$

Now the assertion follows by letting $\varepsilon$ tend to 0 .

Next we turn to deriving a lower bound for the invariance entropy with arbitrary admissible families. The following definition introduces rates of expansion for a semigroup action.

Definition 2.5. Consider a semigroup action $\varphi: M \times S \rightarrow M$ with regular system $\left\{A_{\tau}\right\}_{\tau \geq 0}$. Let $Q \subset M$ be a weakly almost invariant set and $K \subset Q$ a compact subset. Let $\mu$ be a finite Borel measure on an $\varepsilon$-neighborhood $Q_{\varepsilon}=\{x \in M ; d(x, Q)<\varepsilon\}$ of $Q$. The rate of expansion of $\varphi$ with respect to $\left(Q_{\varepsilon}, \mu\right)$ is at least $E$ if for every Borel subset $D \subset K$ and every admissible family $\gamma:[0, \tau] \rightarrow S$ such that $D \cdot \gamma([0, \tau]) \subset Q_{\varepsilon}$, we have that $\mu(D \cdot \gamma(t)) \geq \exp (E t) \cdot \mu(D)$ for every $t \in[0, \tau]$.

Theorem 2.6. Suppose that we are in the setting of Definition 2.5 and let $K \subset Q$ be a compact subset with $\mu(K)>0$. Assume that the rate of expansion of $\varphi$ with respect to $\left(Q_{\varepsilon}, \mu\right)$ is at least $E$. Then

$$
h_{\text {inv }}^{S}(K, Q) \geq \max \{0, E\} .
$$

Proof. Abbreviate $r:=r_{i n v}^{S}(\tau, \varepsilon, K, Q)$. Let $\left\{\gamma_{i}:[0, \tau] \rightarrow S, i=1, \ldots, r\right\}$ be a minimal $(\tau, \varepsilon, K, Q)$-spanning set. Then the sets $U_{i}:=\left\{x \in K, x \cdot \gamma_{i}([0, \tau]) \subset Q_{\varepsilon}\right\}$ form an open cover of $K$. Then one can construct a partition $\left\{D_{i}\right\}_{i=1, \ldots, r}$ of $K$ by Borel subsets $D_{i} \subset U_{i}$. By hypothesis we have that $\mu\left(D_{i} \cdot \gamma_{i}(t)\right) \geq \exp (t E) \cdot \mu\left(D_{i}\right)$ for every $t \in[0, \tau]$. Observe that

$$
r \cdot \mu\left(Q_{\varepsilon}\right) \geq \sum_{i=1}^{r} \mu\left(D_{i} \cdot \gamma_{i}(t)\right) \geq \exp (t E) \cdot \mu(K),
$$


implying

Then it follows that

$$
r_{i n v}^{S}(\tau, \varepsilon, K, Q)=r \geq \frac{\exp (\tau E) \cdot \mu(K)}{\mu\left(Q_{\varepsilon}\right)} .
$$

$$
\limsup _{\tau \rightarrow \infty} \frac{1}{\tau} \log r_{i n v}^{S}(\tau, \varepsilon, K, Q) \geq \limsup _{\tau \rightarrow \infty} \frac{1}{\tau}\left[\tau E+\log \mu(K)-\log \mu\left(Q_{\varepsilon}\right)\right]=E,
$$

and hence

$$
h_{i n v}^{S}(K, Q)=\lim _{\varepsilon \rightarrow 0} \limsup _{\tau \rightarrow \infty} \frac{1}{\tau} \log r_{i n v}^{S}(\tau, \varepsilon, K, Q) \geq E .
$$

\section{CONTROLled INVARIANCE AND INVARIANCE ENTROPY FOR CONTROL SYSTEMS}

This section shows that the invariance entropy introduced above coincides with the invariance entropy for controlled almost invariant sets considered in the context of control systems. The difficulty lies in the fact that the definitions of weak almost invariance and of invariance entropy given in Definitions 1.7 and 1.10, respectively, consider arbitrary admissible families, not just admissible families generated by control functions.

For simplicity, we consider controls given by

$$
\mathcal{U}:=\{u: \mathbb{R} \rightarrow U, \text { locally integrable }\}
$$

with a subset $U \subset \mathbb{R}^{m}$. Let $M$ be a Riemannian manifold. Controlled almost invariance of control systems is defined as follows.

Definition 3.1. For a control system $\Sigma$ of the form (1.2) a compact subset $Q \subset M$ is called controlled almost invariant if for all $x \in Q, \varepsilon, \tau>0$ there is a control $u \in \mathcal{U}$ such that the corresponding solution satisfies $d(\varphi(t, x, u), Q)<\varepsilon$ for every $t \in[0, \tau]$.

Invariance entropy in this context is defined as follows (cf. Colonius and Kawan [6]).

Definition 3.2. Let $K$ be a compact subset of a controlled almost invariant set $Q \subset M$. For $\varepsilon, \tau>0$ a $(\tau, \varepsilon, K, Q)$-spanning set is a set $\mathcal{R} \subset \mathcal{U}$ of control functions such that for every $x \in K$ there is $u \in \mathcal{R}$ with $d(\varphi(t, x, u), Q)<\varepsilon$ for all $t \in[0, \tau]$. Denote the minimal cardinality of a $(\tau, \varepsilon, K, Q)$-spanning set by $r^{\Sigma}(\tau, \varepsilon, K, Q)$ and let

$$
h^{\Sigma}(\varepsilon, K, Q):=\limsup _{\tau \rightarrow \infty} \frac{1}{\tau} \log r^{\Sigma}(\tau, \varepsilon, K, Q) .
$$

The invariance entropy is defined by

$$
h_{i n v}^{\Sigma}(K, Q):=\lim _{\varepsilon \rightarrow 0} h^{\Sigma}(\varepsilon, K, Q) .
$$

Note that here spanning sets are determined by controls, not by admissible families.

Example 3.3. Suppose that system (1.2) is control-affine; i.e., it has the form

$$
\dot{x}=f_{0}(x)+\sum_{i=1}^{m} u_{i}(t) f_{i}(x), u=\left(u_{i}\right) \in \mathcal{U},
$$


with compact and convex control range $U$. If the system is locally accessible and $D$ is a bounded control set, then $Q:=\operatorname{cl} D=\operatorname{cl}(\operatorname{int} D)$ is controlled invariant; cf. Colonius and Kliemann [5, Lemma 3.2.13(i) and Proposition 4.3.3].

We begin our analysis with the following crucial lemma relating arbitrary admissible families to trajectories corresponding to a control $u$.

Lemma 3.4. Consider control system (1.2) with semigroup $S$ and regular system $\left(A_{\tau}\right)_{\tau \geq 0}$ given by (1.3) and (1.4), respectively. Let $\tau, C>0$ and let $N \subset M$ be an open subset such that, for every $x \in M$ and $u \in \mathcal{U}$ with $\varphi(t, x, u) \in N$ for all $t \in[0, \tau]$, the trajectory satisfies

$$
d\left(\varphi\left(t_{1}, x, u\right), \varphi\left(t_{2}, x, u\right)\right) \leq C\left|t_{1}-t_{2}\right| \text { for all } t_{1}, t_{2} \in[0, \tau] .
$$

Let $\gamma:[0, \tau] \rightarrow S$ be an admissible family and let $K \subset N$ be a compact set such that $x \cdot \gamma([0, \tau]) \subset N$ for every $x \in K$. Then for every $\eta>0$ and every $x \in K$ there exists a control $\tilde{u} \in \mathcal{U}$ with

$$
d(x \cdot \gamma(t), \varphi(t, x, \tilde{u}))<\eta \text { for all } t \in[0, \tau] .
$$

Proof. Observe that $K \cdot \gamma([0, \tau]) \subset N$ is a compact subset and we can take $\eta<$ $d(K \cdot \gamma([0, \tau]), M \backslash N)$. Analogously, observe that there exists a $\delta_{1}>0$ such that $\left|t_{1}-t_{2}\right|<\delta_{1}$ implies $d\left(x_{0} \cdot \gamma\left(t_{1}\right), x_{0} \cdot \gamma\left(t_{2}\right)\right)<\eta / 2$ for every $x_{0} \in K$. Property (3.1) shows that if $\varphi\left(t, x_{0}, u\right) \in N$ for $t \in[0, \tau]$ and $t_{1}, t_{2} \in[0, \tau]$ with $\left|t_{1}-t_{2}\right|<\eta /(2 C)$, then

$$
d\left(\varphi\left(t_{1}, x_{0}, u\right), \varphi\left(t_{2}, x_{0}, u\right)\right)<\frac{\eta}{2} .
$$

Let $k \in \mathbb{N}$ be such that $\tau / k \leq \min \left(\delta_{1}, \eta /(2 C)\right)$. We will inductively construct a control $\tilde{u}:[0, \tau] \rightarrow U$ in $\mathcal{U}$ such that

$$
x_{0} \cdot \gamma(i \tau / k)=\varphi\left(i \tau / k, x_{0}, \tilde{u}\right)
$$

for every $i=1, \ldots, k$. Since $\gamma$ is an admissible family, property (ii) in Definition 1.2 shows that $\gamma(\tau / k) \in A_{\tau / k} \backslash A_{<\tau / k}$, and there is $s_{1} \in A_{\tau / k} \backslash A_{<\tau / k}$ with

$$
\gamma(0) \cdot s_{1}=\gamma(0+\tau / k) \text {. }
$$

Using the fact that the regular system is defined by (1.4), one finds a control $u_{1} \in \mathcal{U}$ such that $s_{1}=\left(\tau / k, \varphi\left(\tau / k, \cdot, u_{1}\right)\right)$ and hence

$$
x_{1}:=x_{0} \cdot \gamma(\tau / k)=x_{0} \cdot s_{1}=x_{0} \cdot\left(\tau / k, \varphi\left(\tau / k, \cdot, u_{1}\right)\right)=\varphi\left(\tau / k, x_{0}, u_{1}\right) .
$$

Observe that the curve $\varphi\left(t, x_{0}, u_{1}\right), t \in[0, \tau / k]$, remains inside $N$ due to (3.1), the choice of $k$ and the range of variation of $t$. Proceeding inductively, let

$$
x_{i}:=x_{0} \cdot \gamma(i \tau / k) \in M
$$

for $i \in\{1, \ldots, k-1\}$. One finds an element $s_{i} \in A_{\tau / k} \backslash A_{<\tau / k}$ with

$$
\gamma((i+1) \tau / k)=\gamma(i \tau / k) \cdot s_{i} .
$$

Thus there is a control $u_{i+1} \in \mathcal{U}$ with $s_{i}=\left(\tau / k, \varphi\left(\tau / k, \cdot, u_{i+1}\right)\right)$ satisfying

$$
\begin{aligned}
x_{i+1} & :=x_{0} \cdot \gamma((i+1) \tau / k)=x_{0} \cdot \gamma(i \tau / k) \cdot\left(\tau / k, \varphi\left(\tau / k, \cdot, u_{i+1}\right)\right) \\
& =x_{i} \cdot\left(\tau / k, \varphi\left(\tau / k, \cdot, u_{i+1}\right)\right) \\
& =\varphi\left(\tau / k, x_{i}, u_{i+1}\right) .
\end{aligned}
$$

Now define a control $u \in \mathcal{U}$ by

$$
u(t)=u_{i+1}(t-i \tau / k) \text { for } t \in[i \tau / k,(i+1) \tau / k), i=0,1, \ldots, k-1 .
$$


By construction, the corresponding trajectory $\varphi(t, x, u), t \in[0, \tau]$, satisfies (3.2). Finally observe that for $t \in[0, \tau]$ with $i \tau / k \leq t \leq(i+1) \tau / k$,

$$
\begin{aligned}
& d\left(x_{0} \cdot \gamma(t), \varphi\left(t, x_{0}, u\right)\right) \\
& \leq d\left(x_{0} \cdot \gamma(t), x_{0} \cdot \gamma(i \tau / k)\right)+d\left(x_{0} \cdot \gamma(i \tau / k), \varphi\left(i \tau / k, x_{0}, u\right)\right) \\
& \quad+d\left(\varphi\left(i \tau / k, x_{0}, u\right), \varphi\left(t, x_{0}, u\right)\right) \\
& <\frac{\eta}{2}+0+\frac{\eta}{2}=\eta .
\end{aligned}
$$

The next theorem shows that for control systems, weak almost invariance (defined via admissible families) is equivalent to controlled almost invariance.

Theorem 3.5. Consider control system (1.2) with semigroup $S$ and regular system $\left(A_{\tau}\right)_{\tau \geq 0}$ given by (1.3) and (1.4). Consider a compact subset $Q \subset M$ and $\varepsilon_{0}>0$. Suppose that for every $\tau>0$ and every trajectory with $\sup _{t \in[0, \tau]} d(\varphi(t, x, u), Q)<\varepsilon_{0}$ there exists a constant $C>0$ such that

$$
d\left(\varphi\left(t_{1}, x, u\right), \varphi\left(t_{2}, x, u\right)\right) \leq C\left|t_{1}-t_{2}\right|, \quad 0 \leq t_{1}, t_{2} \leq \tau .
$$

Then $Q$ is weakly almost invariant iff it is controlled almost invariant.

Proof. Clearly, every controlled almost invariant set is weakly almost invariant. Conversely, suppose that $Q$ is weakly almost invariant. Consider $x \in Q, \tau>0$ and $\varepsilon \in\left(0, \varepsilon_{0}\right)$. There exists an admissible family $\gamma$ such that $d(x \cdot \gamma(t), Q)<\varepsilon / 2$ for every $t \in[0, \tau]$. Lemma 3.4 states that there exists a control $u \in \mathcal{U}$ such that $d(\varphi(t, x, u), x \cdot \gamma(t))<\varepsilon / 2$ for every $t \in[0, \tau]$. Thus $d(\varphi(t, x, u), Q)<\varepsilon$ for every $t \in[0, \tau]$ and $Q$ is controlled almost invariant.

Remark 3.6. Observe that inequality (3.3) provides a very mild restriction on (1.2). For instance, if $\|f(x, u)\| \leq C$ for every $u \in U$ and every $x \in Q$, then (3.3) is satisfied.

Next we turn to analyzing invariance entropy. The following lemma will be used to show that a $(\tau, \varepsilon, K, Q)$-spanning set remains a $(\tau, \varepsilon, K, Q)$-spanning set under small deformations. It is a special case of a general result about shrinking of covers; see, e.g., Engelking [7, Theorem 7.1.5]. We include the short proof for the reader's convenience.

Lemma 3.7. Let $\left\{O_{\lambda}\right\}_{\lambda \in \Lambda}$ be an open cover of a compact metric space $(M, d)$. Given $a \varepsilon>0$, define

$$
O_{\lambda}(-\varepsilon):=\left\{x \in M, d\left(x, M \backslash O_{\lambda}\right)>\varepsilon\right\} .
$$

Then there exists $\varepsilon>0$ such that $\left\{O_{\lambda}(-\varepsilon)\right\}_{\lambda \in \Lambda}$ is still an open cover of $M$.

Proof. Since $M$ is compact, it is enough to prove the lemma for a finite open cover $\left\{O_{i}\right\}_{i=1, \ldots, k}$. We claim that there exists $\varepsilon_{1}>0$ such that $\left\{O_{1}\left(-\varepsilon_{1}\right)\right\} \cup\left\{O_{i}\right\}_{i=2, \ldots, k}$ is an open cover of $M$. In fact, notice that $C_{1}:=M \backslash \bigcup_{i=2, \ldots, k} O_{i} \subset O_{1}$ is a compact subset of $M$ and that $D_{1}:=M \backslash O_{1}$ is also compact. We have that $d\left(C_{1}, D_{1}\right)>0$ because $C_{1} \cap D_{1}=\emptyset$. For $\varepsilon_{1}:=d\left(C_{1}, D_{1}\right) / 2$ it follows that $O_{1}\left(-\varepsilon_{1}\right) \supset C_{1}$ and $\left\{O_{1}\left(-\varepsilon_{1}\right)\right\} \cup\left\{O_{i}\right\}_{i=2, \ldots, k}$ is an open cover of $M$. Now consider the open cover $\left\{O_{1}\left(-\varepsilon_{1}\right)\right\} \cup\left\{O_{i}\right\}_{i=2, \ldots, k}$, and find a $\varepsilon_{2}>0$ such that $\left\{O_{1}\left(-\varepsilon_{1}\right)\right\} \cup\left\{O_{2}\left(-\varepsilon_{2}\right)\right\} \cup$ $\left\{O_{i}\right\}_{i=3, \ldots, k}$ covers $M$. Repeating this construction, one ends up with an open cover given by $\left\{O_{i}\left(-\varepsilon_{i}\right)\right\}_{i=1, \ldots, k}$. The assertion follows with $\varepsilon:=\min _{i=1, \ldots, k} \varepsilon_{i}$. 
The next theorem shows that for control systems, the invariance entropy for the associated semigroup action coincides with the invariance entropy for control functions given in Definition 3.2. Thus the notion of admissible families and the associated invariance entropy, which appear adequate in the context of semigroup actions, are natural generalizations from the situation of control systems.

Theorem 3.8. Consider control system (1.2) and the associated semigroup action given by (1.3) and (1.4). Fix compact sets $K \subset Q \subset M$ and suppose that $Q$ is controlled almost invariant. Assume that for every $\tau>0$ there are constants $C, \varepsilon_{0}>0$ such that (3.3) holds for every trajectory with $\sup _{t \in[0, \tau]} d(\varphi(t, x, u), Q)<$ $\varepsilon_{0}$. Then $Q$ is weakly almost invariant for the semigroup action and

$$
h_{i n v}^{S}(K, Q)=h_{i n v}^{\Sigma}(K, Q) .
$$

Proof. The inequality $h_{\text {inv }}^{S}(K, Q) \leq h_{\text {inv }}^{\Sigma}(K, Q)$ holds, because $t \mapsto(t, \varphi(t, \cdot, u))$ is an admissible family for every $u \in \mathcal{U}$. Therefore $r_{i n v}^{S}(\tau, \varepsilon, K, Q) \leq r_{\text {inv }}^{\Sigma}(\tau, \varepsilon, K, Q)$ for every $\tau, \varepsilon>0$, and the inequality follows.

In order to prove the converse inequality, let $\left\{\gamma_{i}\right\}_{i=1, \ldots, k}$ be a minimal $(\tau, \varepsilon, K, Q)$ spanning set of admissible families for the semigroup action (1.3). Set $O_{i}:=\{x \in K$, $d\left(x \cdot \gamma_{i}(t), Q\right)<\varepsilon$ for every $\left.t \in[0, \tau]\right\}$. Then $\left\{O_{i}\right\}_{i=1, \ldots, k}$ is an open cover of $K$. By Lemma 3.7, there is $\eta>0$ such that $\left\{O_{i}(-\eta)\right\}_{i=1, \ldots, k}$ is also an open cover of $K$. The family $\left\{\overline{O_{i}(-\eta)}\right\}_{i=1, \ldots, k}$ also covers $K$ and we may assume that it consists of compact sets. Then

$$
P:=\left\{x \cdot \gamma_{i}(t), x \in \overline{O_{i}(-\eta)} \text { and } t \in[0, \tau] \text { for some } i=1, \ldots, k\right\}
$$

is a compact subset of $\mathbf{N}_{\varepsilon}(Q)=\{y \in M, d(y, Q)<\varepsilon\}$. Set

$$
\beta:=d\left(M \backslash \mathbf{N}_{\varepsilon}(Q), P\right)>0 .
$$

Using Lemma 3.4, for every $\gamma_{i}$, there exist $u_{i} \in \mathcal{U}$ such that

$$
d\left(x \cdot \gamma_{i}(t), \varphi\left(t, x, u_{i}\right)\right)<\beta
$$

for every $x \in \overline{O_{i}(-\eta)}$ and every $t \in[0, \tau]$, implying $\varphi\left(t, x, u_{i}\right) \in \mathbf{N}_{\varepsilon}(Q)$. Therefore $\left\{u_{1}, \ldots, u_{k}\right\} \subset \mathcal{U}$ is a $(\tau, \varepsilon, K, Q)$-spanning set for control system (1.2). It follows that $r_{i n v}^{\Sigma}(\tau, \varepsilon, K, Q) \leq r_{i n v}^{S}(\tau, \varepsilon, K, Q)$ and hence $h_{i n v}^{\Sigma}(K, Q) \leq h_{i n v}^{S}(K, Q)$.

\section{REFERENCES}

[1] R. L. Adler, A. G. Konheim, and M. H. McAndrew, Topological entropy, Trans. Amer. Math. Soc. 114 (1965), 309-319. MR.0175106 (30 \#5291)

[2] Andrei A. Agrachev and Yuri L. Sachkov, Control theory from the geometric viewpoint, Encyclopaedia of Mathematical Sciences, vol. 87, Control Theory and Optimization, II, SpringerVerlag, Berlin, 2004. MR2062547 (2005b:93002)

[3] Victor Ayala, Fritz Colonius, and Wolfgang Kliemann, On topological equivalence of linear flows with applications to bilinear control systems, J. Dyn. Control Syst. 13 (2007), no. 3, 337-362, DOI 10.1007/s10883-007-9021-9. MR2337281(2009k:37035)

[4] Laurent Baratchart, Monique Chyba, and Jean-Baptiste Pomet, A Grobman-Hartman theorem for control systems, J. Dynam. Differential Equations 19 (2007), no. 1, 75-107, DOI 10.1007/s10884-006-9014-5. MR2279947 (2007i:93020)

[5] Fritz Colonius and Wolfgang Kliemann, The dynamics of control, with an appendix by Lars Grüne. Systems \& Control: Foundations \& Applications, Birkhäuser Boston Inc., Boston, MA, 2000. MR1752730(2001e:93001)

[6] Fritz Colonius and Christoph Kawan, Invariance entropy for control systems, SIAM J. Control Optim. 48 (2009), no. 3, 1701-1721, DOI 10.1137/080713902. MR2516184 (2010f:37155) 
[7] Ryszard Engelking, General topology, translated from the Polish by the author. Monografie Matematyczne, Tom 60 [Mathematical Monographs, Vol. 60], PWN-Polish Scientific Publishers, Warsaw, 1977. MR0500780 (58 \#18316b)

[8] K. H. Hofmann and L. N. Stojanov, Topological entropy of group and semigroup actions, Adv. Math. 115 (1995), no. 1, 54-98, DOI 10.1006/aima.1995.1050. MR.1351326 (97a:22006)

[9] Velimir Jurdjevic, Geometric control theory, Cambridge Studies in Advanced Mathematics, vol. 52, Cambridge University Press, Cambridge, 1997. MR 1425878 (98a:93002)

[10] Anatole Katok and Boris Hasselblatt, Introduction to the modern theory of dynamical systems, with a supplementary chapter by Katok and Leonardo Mendoza. Encyclopedia of Mathematics and its Applications, vol. 54, Cambridge University Press, Cambridge, 1995. MR 1326374 (96c:58055)

[11] Christoph Kawan, Invariance entropy of control sets, SIAM J. Control Optim. 49 (2011), no. 2, 732-751, DOI 10.1137/100783340. MR2801217(2012d:93074)

[12] C. Kawan, Lower bounds for the strict invariance entropy, Nonlinearity 24 (2011), 1909-1935. MR2805586(2012d:37206)

[13] Christoph Kawan, Upper and lower estimates for invariance entropy, Discrete Contin. Dyn. Syst.-A 30 (2011), 169-186. MR.2773136 (2012c:93041)

[14] J. R. Munkres, Topology, Prentice Hall, 2000.

[15] Girish N. Nair, Robin J. Evans, Iven M. Y. Mareels, and William Moran, Topological feedback entropy and nonlinear stabilization, IEEE Trans. Automat. Control 49 (2004), no. 9, 15851597, DOI 10.1109/TAC.2004.834105. MR2086921(2005e:94050)

[16] Mauro Patrão, Entropy and its variational principle for non-compact metric spaces, Ergodic Theory Dynam. Systems 30 (2010), no. 5, 1529-1542, DOI 10.1017/S0143385709000674. MR:2718907(2011m:37017)

[17] Luiz San Martin, Invariant control sets on flag manifolds, Math. Control Signals Systems 6 (1993), no. 1, 41-61, DOI 10.1007/BF01213469. MR1358060 (96i:93020)

Institut für Mathematik, Universität Augsburg, Augsburg, Germany

Departamento de Matemática, Universidade Estadual de Maringá, Maringá, Brazil

Departamento de Matemática, Universidade Estadual de Maringá, Maringá, Brazil 